\title{
Volvulus and Under Appreciated Complication of Mixed Connective Tissue Disease: A Case Report
}

\author{
Christina M. Bortz ${ }^{\mathrm{a}}$, Amy Yu ${ }^{\mathrm{a}}$, David J. Ozeri ${ }^{\mathrm{b}, \mathrm{c}}$
}

\begin{abstract}
We report a case of volvulus in mixed connective tissue disease (MCTD). To our knowledge, this is the first reported case of volvulus in a patient with MCTD. MCTD is characterized by high levels of anti-U1 ribonucleoprotein (anti-U1 RNP) plus overlapping features of systemic lupus erythematosus (SLE), systemic sclerosis (SSc), polymyositis and dermatomyositis. Gastrointestinal (GI) symptoms in MCTD are common, but are usually limited to disordered motility in the upper GI tract. Volvulus is frequently reported as a complication of SSc, but should also be considered in MCTD.
\end{abstract}

Keywords: MCTD; Volvulus; Raynaud's; Anti-RNP

\section{Introduction}

Mixed connective tissue disease (MCTD) was introduced as a distinct autoimmune rheumatologic disease by Sharp et al in 1972 [1]. MCTD is characterized by high levels of anti-U1 ribonucleoprotein (anti-U1 RNP) in addition to characteristic overlapping features of systemic lupus erythematosus (SLE), systemic sclerosis ( $\mathrm{SSc}$ ), polymyositis and dermatomyositis [2]. Gastrointestinal (GI) symptoms in MCTD are common, but are usually limited to disordered motility in the upper GI tract [3]. Volvulus related to a rheumatological disease is most commonly seen in patients with SSc. To our knowledge, this is the first reported case of volvulus in a patient with MCTD.

\section{Case Report}

A 69-year-old female with a past medical history of SLE,

Manuscript submitted February 15, 2018, accepted March 16, 2018

aDepartment of Medicine, Brooklyn Methodist Hospital, 506 6th St. Brooklyn, NY 11215, USA

bDivision of Rheumatology, Department of Medicine, Brooklyn Methodist Hospital, 506 6th St. Brooklyn, NY 11215, USA

${ }^{\mathrm{c} C}$ Corresponding Author: David J. Ozeri, Division of Rheumatology, Department of Medicine, Brooklyn Methodist Hospital, 506 6th St. Brooklyn, NY 11215, USA. Email: djo9005@nyp.org

doi: https://doi.org/10.14740/jmc3014w pulmonary hypertension, and pulmonary fibrosis presented with right upper quadrant (RUQ) pain, bloody bowel movements, and multiple episodes of vomiting for three days. She described her pain as cramping and sharp in nature, non-radiating, and intermittent throughout the day. She had no history of previous abdominal surgery. On physical exam, the abdomen was soft and slightly distended with tenderness in the RUQ without rebound or guarding. Labs were significant for WBC $18,300 / \mu \mathrm{L}$ and $\mathrm{Hgb} 12.2 \mathrm{~g} / \mathrm{dL}$; otherwise, $\mathrm{CBC}$ and BMP were unremarkable. CT of the abdomen revealed a dilated right colon with a twist in the RUQ, consistent with cecal volvulus (Fig. 1). The patient was taken for emergent exploratory laparotomy where a right hemicolectomy was performed with ileocolic anastomosis.

Rheumatology was consulted postoperatively for optimization of the patient's SLE. She had carried the diagnosis for 20 years and was maintained on hydroxychloroquine and prednisone. Her main complaints were dysphagia and episodic attacks of pain in hands and feet associated with cold temperatures. She denied any history of malar rash, photosensitivity, oral ulcers or joint pain. On physical examination, the patient was noted to have sclerodactyly, evidence of Raynaud's phenomenon and decreased oral aperture.

Based on clinical history and physical examination, there was high suspicion for SSc. Serologies revealed negative anticentromere, anti-Scl 70, anti-Smith, and anti-dsDNA antibodies. Anti-U1 RNP was elevated, consistent with the diagnosis of MCTD.

\section{Discussion}

Our case demonstrates the significant overlap between collagen vascular diseases that may lead to misdiagnosis. Clinical manifestations of MCTD, such as generalized malaise, arthralgia, myalgia, and low-grade fevers, are non-specific and similar to those seen in SLE and SSc. Involvement of the upper GI tract is another common overlap symptom, seen in about $60-80 \%$ of patients with SSc and MCTD. Four features that strongly suggest a diagnosis of MCTD are Raynaud phenomenon, absence of renal and CNS disease, pulmonary hypertension, and anti-U1 RNP [2].

The entire GI tract, from the oral cavity to the anus, may be affected in collagen vascular disease. The primary issue is dysmotility of the smooth muscle wall, which presents with a wide array of symptoms, including nausea, vomiting, diarrhea with alternating constipation, as well as fecal incontinence [4]. 

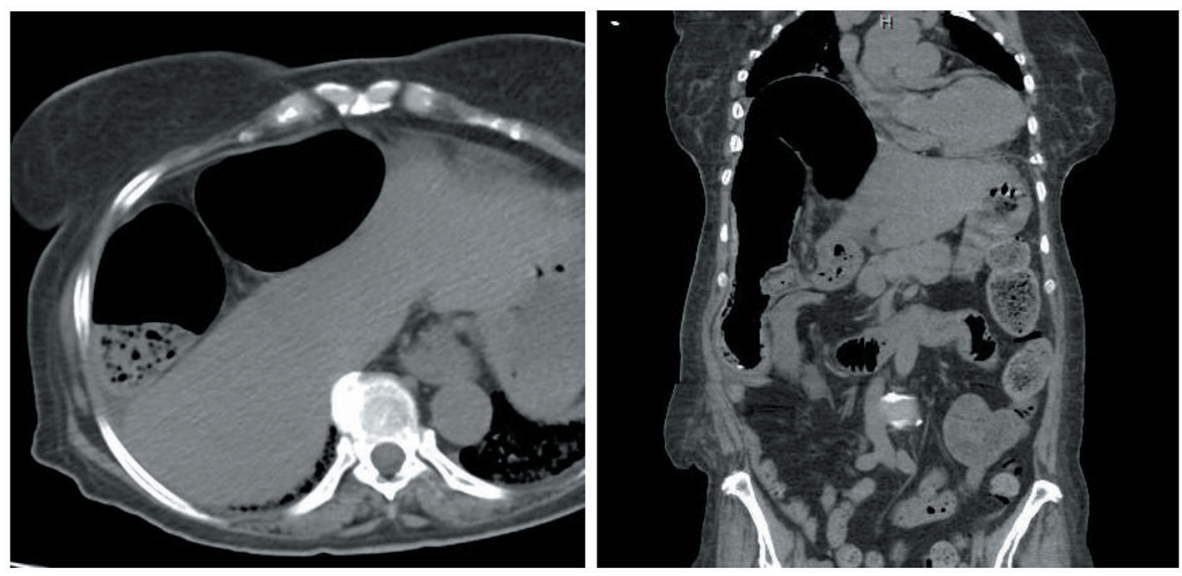

Figure 1. CT of abdomen showing right colonic dilatation in the coronal and transverse views suggestive of volvulus.

When persistent and severe, malabsorption occurs, with subsequent weight loss and malnutrition $[5,6]$. The esophagus is most commonly involved, affecting $80 \%$ of patients with collagen vascular disease. Esophageal dysmotility leads to an abnormal peristaltic wave as well as patency of lower esophageal sphincter, which manifests as gastroesophageal reflux disease (GERD). Chronic acid exposure increases the risk of esophageal strictures and Barrett's esophagus. In the stomach, dysmotility manifests as gastroparesis, while in the small bowel pseudo-obstruction occurs from stasis of digestive contents, leading to dilation and subsequent bacterial overgrowth. There is rarely a mechanical blockage, and symptoms improve with conservative management [5].

Volvulus in rheumatologic disorders is most commonly associated with SSc [4]. Although the mechanism is not fully understood, colonic histologic findings show tissue deposition of $\mathrm{IgG}$ and $\mathrm{C} 3$, suggesting that volvulus in SSc is due to collagen deposition and chronic inflammation that results in muscle atrophy, fibrosis, and eventual disordered contractility and motility of the intestinal smooth muscle wall [4]. This is the same histologic pathway that leads to the esophageal dysmotility seen in MCTD. Thus, although there has never been a reported case of volvulus in a patient with MCTD, it is likely that the pathophysiology is the same. Recognizing overlap features of collagen vascular diseases to form an accurate diagnosis is vital in establishing proper treatment and prognosis.

\section{References}

1. Sharp GC, Irvin WS, Tan EM, Gould RG, Holman HR. Mixed connective tissue disease - an apparently distinct rheumatic disease syndrome associated with a specific antibody to an extractable nuclear antigen (ENA). Am J Med. 1972;52(2):148-159.

2. Bennett RM, O’Connell DJ. Mixed connective tisssue disease: a clinicopathologic study of 20 cases. Semin Arthritis Rheum. 1980;10(1):25-51.

3. Ciang NC, Pereira N, Isenberg DA. Mixed connective tissue disease-enigma variations? Rheumatology (Oxford). 2017;56(3):326-333.

4. Haque U, Yardley J, Talamini M, Wigley F. Colon stricture and volvulus in a patients with scleroderma. J Rheumatol. 1999;26(10):2268-2272.

5. Nagaraja V, McMahan ZH, Getzug T, Khanna D. Management of gastrointestinal involvement in scleroderma. Curr Treatm Opt Rheumatol. 2015;1(1):82-105.

6. Shreiner AB, Khanna D. Gastrointestinal involvement in systemic sclerosis. The Digestive Involvement in Systemic Autoimmune Diseases Handbook of Systemic Autoimmune Diseases, 2017:243-261. 